\title{
A Mathematical Model for Performance Analysis of IEEE 802.15.4 Non-Beacon Enabled Mode
}

\author{
Chiara Buratti \\ WiLAB, DEIS \\ University of Bologna \\ Italy \\ Email: c.buratti@unibo.it
}

\author{
Roberto Verdone \\ WiLAB, DEIS \\ University of Bologna \\ Italy \\ Email: roberto.verdone@unibo.it
}

\begin{abstract}
In this paper a mathematical model for the NonBeacon Enabled Mode of the IEEE 802.15.4 Multiple Access Control (MAC) protocol, is provided. A Wireless Sensor Network (WSN) composed of nodes which transmit data to a sink through direct links, is considered. Upon reception of a query from the sink, the nodes transmit their packets by using the Carrier Sensing Multiple Access with Collision Avoidance algorithm defined by IEEE 802.15.4. The model allows the evaluation of the statistical distribution of the traffic generated by the nodes, the success probability for the transmission of a packet, and the mean energy spent by a node for a transmission. The results are validated through simulations. The model differs from those previously published by other Authors in the literature as it precisely follows the MAC procedure defined by the standard, in the context of the WSN scenario described above. Performance obtained by varying the parameters of the backoff algorithm, are evaluated and compared. Results show that a tradeoff between energy efficiency and reliability is needed.
\end{abstract}

\section{INTRODUCTION}

IEEE 802.15.4 [1] is a short-range wireless technology specifically devised to support low power, low cost, low data rate Personal Area Networks (PANs). The main field of application of this technology is the implementation of Wireless Sensor Networks (WSNs): systems with tiny devices deployed in an area and monitoring physical phenomena, reporting to one or more sinks the information achieved through wireless links, upon reception of queries, or detection of events [2], [3]. The standard defines the physical and Multiple Access Control (MAC) aspects. The protocols of the higher layers are specified by the ZigBee Alliance [4]. The 802.15.4 standard allows two types of channel access mechanisms: beacon or non-beacon enabled, using a slotted or unslotted Carrier Sensing Multiple Access with Collision Avoidance (CSMA/CA) protocol, respectively. Concerning network topology, two basic options are allowed: stars, formed around a node acting as a coordinator, and the peer-to-peer topology, where each device is able to form multiple direct links to other devices. Star topologies are preferable when the PAN area is small; in this case, communication is controlled by the PAN coordinator. The use of beacon or non-beacon enabled mode depends on the application: beacon transmissions are disadvantageous when no periodic or frequent messages are expected from the coordinator, and only sporadic traffic is transmitted by network devices. The number of nodes associated to a coordinator when a star topology is used, ranges from 2 to 10 as is widely accepted that 802.15.4 does not support larger network sizes in this case [5].

In this paper, we consider a WSN composed of a small number of devices (hereafter denoted as nodes); each node upon reception of a query from the PAN coordinator (denoted as sink in the following), takes one sample of a given phenomenon (e.g. atmospheric pressure, or temperature) and forwards it through a direct link to the sink. The 802.15.4 nonbeacon enabled mode is used. The nodes compete to access the channel, in order to transmit the data required. Once transmission is performed, they move to an idle state, till the next query is received. The interval between two successive queries is denoted as round. It is worthwhile mentioning that such scenario can also be considered as part of a larger network where the PAN coordinators are cluster-heads coordinating specific clusters of nodes through the non-beacon enabled mode.

Given the above scenario, the aim of this paper is to provide an analytical model for the description of the transitions between node states (backoff, sensing, transmission, idle) of the CSMA/CA 802.15.4 MAC protocol. The model is then finalized to the derivation of the probability that a generic node succeeds in the access to the channel and in transmitting its packet. These probabilities are evaluated as a function of time, starting from reception of the query, till the end of the round. Moreover, the model allows evaluation of the overall success probability for the transmission of a packet in a round, and the mean energy consumed by a node during a round ${ }^{1}$.

Some papers have been published in the literature, trying to describe analytically the behavior of the 802.15.4 MAC protocol: the majority of them are related to beacon enabled networks [7]-[9], whereas few works deal with the non-beacon enabled mode [10]. Some of these models do not capture

\footnotetext{
${ }^{1}$ Other aspects are considered in [6]
} 
correctly the CSMA/CA protocol and, above all, even if the form of the analysis used is similar (all papers are based on the Bianchi's model for the 802.11 DCF protocol [11]), many differences between these papers and our work, are present. First of all our model predicts the statistical distribution of the traffic generated by the WSN. The statistics are evaluated over time; whereas the works cited above study the asymptotic behavior of the network (i.e., for $t \rightarrow \infty$ ). Moreover, in these works the probability to find the channel busy is evaluated regardless of the backoff stage in which the node is. Instead, our model captures the different probabilities at the different backoff stages.

To validate the mathematical model, comparison to simulations is performed.

The paper is outlined as follows: Section II describes the unslotted 802.15.4 CSMA/CA protocol modelled, Section III introduces assumptions and notations used, whereas in Section IV the analytical model is presented. In Section V the performance metrics are derived and, finally, Sections VI and VII report numerical results and conclusions, respectively.

\section{THE IEEE 802.15.4 MAC PROTOCOL}

We consider here the non-beacon enabled mode of the IEEE802.15.4 MAC protocol [1]. According to the standard, nodes have to use an unslotted CSMA/CA protocol to access the channel and transmit their packets. The algorithm is implemented using units of time called backoff periods, and one backoff period is equal to $20 T_{s}$, where $T_{s}=16 \mu \mathrm{sec}$ is the symbol time duration [1].

Each node maintains two variables for each transmission attempt: $N B$, and $B E . N B$ is the number of time units the CSMA/CA algorithm was required to backoff while attempting the current transmission; this value will be initialized to 0 before each new transmission attempt. $B E$ is the backoff exponent, which is related to the maximum number of backoff periods a node will wait before attempting to assess a channel. $B E$ will be initialized to the value of $B E_{\min }$, and cannot assume a value larger than $B E_{\max }$.

The steps of the CSMA/CA algorithm, are the following. First of all $N B$ and $B E$ are initialized and then the MAC sublayer will delay any activities for a random number of complete backoff periods in the range $\left(0,2^{B E}-1\right)$ [step (1)]. After this delay, channel sensing is performed for one unit of time [step (2)]. If the channel is assessed to be busy [step (3)], the MAC sublayer will increment both $N B$ and $B E$ by one, ensuring that $B E$ is no larger than $B E_{\max }$. If the value of $N B$ is less than or equal to $N B_{\max }$ the CSMA/CA algorithm will return to step (1). If the value of $N B$ is larger than $N B_{\max }$, the CSMA/CA algorithm will terminate with a "Failure", meaning that the node does not succeed in the access to the channel. If the channel is assessed to be idle [step (4)], the MAC sublayer will begin transmission of the frame immediately ("Success" in the access to the channel).

As stated in Section I, performance will be evaluated for different values of $B E_{\min }, B E_{\max }$ and $N B_{\max }$.

\section{Analytical Model: Assumptions And NOTATIONS}

\section{A. Reference Scenario, Model Assumptions}

We consider $N$ nodes in the WSN. We assume that each node transmits a packet of 10 Bytes, which correspond to $20 T_{s}$, at each round. In [6] performance obtained by varying the packet size are considered; here, instead, the packet size is fixed. All nodes start the backoff algorithm at the same time, when the query transmitted by the sink is received (no propagation delay is present; therefore, all nodes receive the packet at the same time, denoted as $t=0$ ). In our model we subdivide the time axis into slots of $20 T_{s}$. This duration, in fact, corresponds to the packet size, the backoff period unit and the duration of the sensing phase.

Ideal channel conditions are assumed: all nodes can "hear" each other and therefore, no hidden terminal problem is taken into consideration. Similar scenarios and assumptions are considered in many papers in the literature [7]-[11]. Collisions between nodes may occur in case two or more nodes perform channel sensing at the same time, find the channel free and transmit simultaneously their packets. For the sake of energy efficiency, no acknowledge and retransmission mechanism are implemented; therefore, when a packet collides it is definitely lost for that round.

\section{B. Node States}

Generally speaking, a node accessing the channel during the round can be in one of four states: backoff, sensing, transmission or idle. However, if after sensing the channel is free, transmission immediately occurs, followed by a sequence of idle states till the end of the round. Thus, given the objectives of this paper, we need to model only the backoff and sensing states.

The node state is modelled as a bidimensional process $\left\{B O_{c}(t), B O_{s}(t)\right\}$, where $B O_{c}(t)$ and $B O_{s}(t)$ represent the backoff time counter and the backoff stage at time $t$, respectively. They are both time-discrete stochastic processes assuming discrete values. Therefore, the process is a chain; however, not a Markovian chain [12] because $B O_{c}(t)$ is not a memoryless process and its value depends on its history (i.e, its value depends on how many times the node has tried to access the channel without success). $t$ is an integer, representing the time slot and, more precisely, the $j$-th slot (from $j \cdot 20 T_{s}$ to $\left.(j+1) \cdot 20 T_{s}\right)$ is denoted by $t=j$.

The initial value of backoff time counter $\left(B O_{c}(0)\right)$ is uniformly distributed in the range $\left[0, W_{N B}-1\right]$, where $W_{N B}=2^{B E}$ is the dimension of the contention window, and $N B \in\left[0, N B_{\max }\right]$. The value of $B E$ depends on the second process characterising the state: $B O_{s}(t)$. We can identify $N B_{\max }+1$ different backoff stages, obtained by considering the different possible combinations of the pair $(N B, B E)$, corresponding to the $N B_{\max }+1$ different values of $W_{N B}$. In Table I, the different backoff stages with the correspondent $W_{N B}$ values (denoted as $W_{0}, . ., W_{N B_{\max }}$ ), are shown. 
In the following, the generic state will be denoted as $Q(t)=$ $\left\{B O_{c}, B O_{s}, t\right\}$ and the probability of being in a generic state will be denoted as $P\left\{B O_{c}=c, B O_{s}=i, t=j\right\}=P\{c, i, j\}$.

TABLE I

THE BACKOFF STAGES.

\begin{tabular}{|c|c|c|c|}
\hline$B O_{s}$ & $N B$ & $B E$ & $W_{N B}=2^{B E}$ \\
\hline 0 & 0 & $B E_{\min }$ & $W_{0}=2^{B E_{\min }}$ \\
1 & 1 & $B E_{\min }+1$ & $W_{1}=2^{B E_{\min }+1}$ \\
$\ddot{.}$ &.. &.$\cdot$ &.. \\
$N B_{\max }$ & $N B_{\max }$ & $B E_{\max }$ & $W_{N B_{\max }}=2^{B E_{\max }}$ \\
\hline
\end{tabular}

\section{Formulation of the Mathematical Model}

\section{A. Steps Followed by the Model}

Let us denote as $p_{b}^{j}$ the probability that in the $j$-th slot the channel is found to be busy after sensing. This probability will be initially left as parameter, and its computation will be performed in Section V-D.

The model provides the following metrics:

1) the probability that a node transmits its packet in a given slot, $j$, denoted as $P\left\{T^{j}\right\}$;

2) the probability that a node transmits its packet with success in a given slot, $j$, denoted as $P\left\{Z^{j}\right\}$;

3) the success probability for a transmission, that is the probability that a generic packet is transmitted with success on the channel in a round whatever the slot, denoted as $p_{s}$;

4) the mean energy spent by a node in a round to transmit its packet, denoted as $E_{\text {mean }}$.

Since there exists a maximum value for $N B$, there will be also a maximum delay that affects a packet. This maximum is reached in case a node extracts at every backoff stage the higher backoff time counter and at the end of each backoff stage it always finds the channel busy. In this case, the node is in backoff state for $\sum_{k=0}^{N B_{\max }}\left(W_{k}-1\right)$ slots and will be sensing the channel for $N B_{\max }+1$ slots. Therefore, the last slot for transmitting is $t_{\max }=\sum_{k=0}^{N B_{\max }} W_{k}$, whereas sensing is possible only for $t=j \in\left[0, t_{\max }-1\right]$.

The probability $P\left\{T^{j}\right\}$ with $j \in\left[0, t_{\max }\right]$ depends on the probability of being in sensing at the previous slot $(j-1)$. To determine the sensing probabilities, we model the behavior of a single node, using a state transition diagram [12], describing the relation between all possible states in which a node can be (Figure 1). From this diagram we obtain the probability of being in sensing at the $j$-th slot and at the $i$-th backoff stage $\left(B O_{s}=i\right)$, denoted as $P\left\{S_{i}^{j}\right\}=P\{0, i, j\}$. This is made in the remainder of this Section. From these probabilities, we can derive the probability of being in sensing at the $j$ th slot, whatever the backoff stage, denoted as $P\left\{C^{j}\right\}$ with $j \in\left[0, t_{\max }-1\right]$, and, therefore, $P\left\{T^{j}\right\}$ (see Section V-A). The probability $p_{s}$ and the mean energy spent, $E_{\text {mean }}$, instead, depend on $P\left\{T^{j}\right\}$ and will be derived in Sections V-B and V-C, respectively.

\section{B. Sensing Probabilities}

The state transition diagram of the bidimensional process $Q(t)$, should be presented through $N B_{\max }+1$ different Figures. Each Figure is related to a value of $\mathrm{BO}_{s}$. For the sake of conciseness we report here only the diagram related to the case $B O_{s}=0$ (Figure 1), and we refer to [6] for the other parts of the chain and for a more exhaustive description. Since the Figure refers to $B O_{s}=0$, for the sake of simplicity in the drawings the generic backoff state (ovals in the Figure) is simply denoted as $\{c, j\}$, omitting the value of $B O_{s}$; the sensing states (squares) are denoted as $S^{j}$ with no subscript $i$.

In the following the state transition diagram related to the first backoff stage (Figure 1) will be described. The probabilities of being in the different states of the chain and the transition probabilities between the states, will be provided.

1) First Backoff Stage $\left(B O_{s}=0\right)$ : At the beginning of the backoff algorithm, each node extracts an integer, uniformly distributed between 0 and $W_{0}-1$. At $t=0$ a node enters, with probability $1 / W_{0}$, one of the states $\{c, 0,0\}$ with $c \in\left[0, W_{0}-\right.$ 1]. If a node extracts 0 , it will sense the channel in slot 0 and it will transmit its packet in slot 1 . In fact, since no transmissions may occur in slot $0\left(P\left\{T^{0}=0\right\}\right)$, the channel will be certainly found free $\left(p_{b}^{0}=0\right)$. If a value larger than 0 is extracted, the node will enter in $\{c, 0,0\}$ with $c \in\left[1, W_{0}-1\right]$ (see Figure 1) and it will decrease its backoff counter at each successive slot (i.e., a node passes from state $\{c, 0,0\}$ to $\{c-1,0,1\}$ with probability 1 ), until the counter will reach the value 0 , when the node will sense the channel. After the sensing phase the node will transmit the packet in case the channel is found free, otherwise it will pass to the following backoff stage and an other backoff counter value, uniformly distributed between 0 and $W_{1}-1$, will be extracted. In Figure 1, the transitions originated by the sensing states enter the states of backoff or sensing of the part of the chain related to $B O_{s}=1$ (not reported here [6]). For example, if a node is in the state $S_{0}^{1}$ and it finds the channel busy, it will enter the state $S_{1}^{2}$, or one of the states $\{c, 1,2\}$, with $c \in\left[0, W_{1}-1\right]$, with the same probability $p_{b}^{1} / W_{1}$. The state of arrival depends on the new backoff counter value extracted.

Denoting as $P\left\{B O_{c}=c_{1}, B O_{s}=i_{1}, t=j_{1} \mid B O_{c}=\right.$ $\left.c_{0}, B O_{s}=i_{0}, t=j_{0}\right\}=P\left\{c_{1}, i_{1}, j_{1} \mid c_{0}, i_{0}, j_{0}\right\}$, the transition probability from the state $\left\{c_{0}, i_{0}, j_{0}\right\}$ to the state $\left\{c_{1}, i_{1}, j_{1}\right\}$, the transition probabilities between the backoff states are given by:

$$
P\{c, 0, j+1 \mid c+1,0, j\}=1,
$$

for $c \in\left[0, W_{0}-2\right]$ and $j \in\left[0, W_{0}-2\right]$. This equation accounts for the fact that, at the beginning of each time slot, the backoff time counter is decreased by 1 with probability 1 , until it reaches the value 0 .

From the chain we can obtain the probabilities of being in a sensing state, given by:

$$
P\left\{S_{0}^{j}\right\}=\frac{1}{W_{0}},
$$

for $j \in\left[0, W_{0}-1\right]$. 
2) Other Backoff Stages $\left(B O_{s}=i\right.$ with $\left.i \in\left[1, N B_{\max }\right]\right)$ : We do not report the Figures related to these backoff stages (the reader can refer to [6]). These Figures are obtained by applying the same rationale used in the chain for $B O_{s}=0$. Here we just report the expressions of the sensing probabilities obtained from the chain. In the following we will denote as $W_{s p} \quad \sum_{k=0}^{i-1} W_{k}$ and $W_{s t} \sum_{k=0}^{i} W_{k} . W_{s p}$ and $W_{s t}$ are the partial and total sum of the contention windows at the current backoff stage, respectively. As in the previous case, the transition probabilities between backoff states are given by:

$$
P\{c, i, j+1 \mid c+1, i, j\}=1,
$$

for $c \in\left[0, W_{i}-2\right]$ and $j \in\left[i+1, W_{s t}-2\right]$.

The transition probabilities between the states of the $(i-1)$-th backoff stage $\left(B O_{s}=i-1\right)$ and the current backoff stage $\left(B O_{s}=i\right)$, are given by:

$$
P\{c, i, j+1 \mid 0, i-1, j\}=\frac{p_{b}^{j}}{W_{i}},
$$

for $c \in\left[0, W_{i}-1\right]$ and $j \in\left[i, W_{s p}-1\right]$. This equation accounts for the fact that in case the channel at the $j$-th slot is found busy (which happens with probability $p_{b}^{j}$ ), the node will enter one of the states $\{c, i, j+1\}$, with $c \in\left[0, W_{i}-1\right]$, with the same probability $1 / W_{i}$. Therefore, the probability that a node coming from the state $\{0, i-1, j\}$ enters the state $\{c, i, j+1\}$, is the product of the probabilities $p_{b}^{j}$ and $1 / W_{i}$.

In case $W_{s p}<i+W_{i}+1$ the probability of being in the sensing states, is given by [6]:

$$
P\left\{S_{i}{ }^{j}\right\}=\left\{\begin{array}{lr}
0 & \text { for } j<i+1 \\
P\left\{S_{i}{ }^{j-1}\right\}+P\left\{S_{i-1}^{j-1}\right\} \cdot \frac{p_{b}{ }^{j-1}}{W_{i}} & \text { for } j \in\left[i+1, W_{s p}\right] \\
P\left\{S_{i}{ }^{W_{s p}}\right\} & \text { for } j \in\left[W_{s p}+1, i+W_{i}\right] \\
P\left\{S_{i}{ }^{j-1}\right\}-P\left\{S_{i-1}^{\left.j-1-W_{i}\right\} \cdot p_{b}{ }^{j-1-W_{i}}}\right. & \\
& \text { for } j \in\left[i+W_{i}+1, W_{s t}-1\right] \\
0 & \text { for } j>=W_{s t}
\end{array}\right.
$$

In case, instead, $W_{s p} \geq i+W_{i}+1$, the probability of being in the sensing states in this case, is given by [6]:

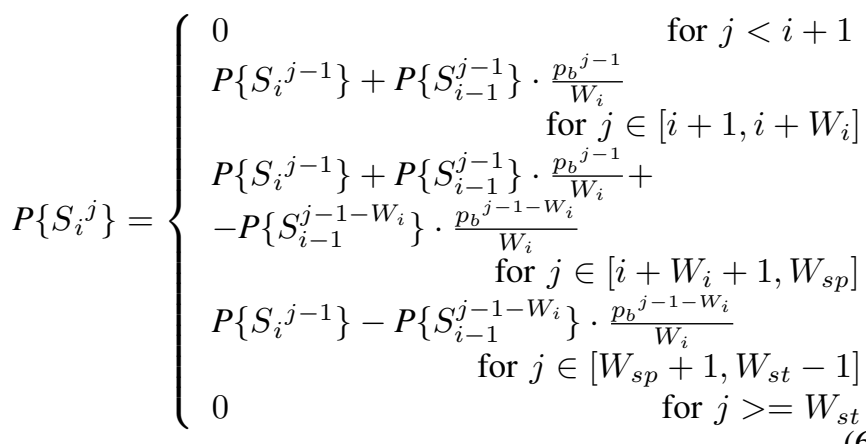

In conclusion, in this Section we have derived the expressions of the sensing probabilities (eqs. (2), (5), (6)) in all possible backoff stages and slots.

\section{Performance Metrics Derived from the Model}

\section{A. Transmission Probabilities}

As stated above, the aim of our model is to evaluate the probability that a generic node transmits a packet in a certain slot $j, P\left\{T^{j}\right\}$, with $j \in\left[0, t_{\max }\right]$.

A node will transmit its packet when, at the end of the backoff algorithm $\left(B O_{c}=0\right)$, it will sense the channel finding it free; therefore the above probability is given by:

$$
P\left\{T^{j}\right\}=P\left\{C^{j-1}\right\} \cdot\left(1-p_{b}^{j-1}\right) .
$$

The probability of being in sensing state at slot $j$, is given by the sum of the probabilities of being in sensing at the $j$-th slot and at the $i$-th backoff stage, considering all the possible backoff stages [6]. Therefore, it is given by:

$$
P\left\{C^{j}\right\}=\sum_{i=0}^{N B_{\max }} P\left\{S_{i}^{j}\right\} .
$$

\section{B. Success Probability}

To evaluate the success probability, we have to model how the number of nodes that compete for the access to the channel, varies with time. We denote as $N_{c}^{j}$ the number of nodes which have not transmitted yet at the end of slot $(j-1)$ and that will compete for slot $j . N_{c}^{j}$ is a random variable, binomially distributed. In general, the probability that $k$ over a number $\hat{N}$ of nodes at slot $j$ have not transmitted the packet yet, conditioned on the fact that at slot $j-1 \hat{N}$ nodes are competing for the channel $\left(N_{c}^{j-1}=\hat{N}\right)$, is given by:

$$
\begin{aligned}
& P\left\{N_{c}^{j}=k \mid N_{c}^{j-1}=\hat{N}\right\}= \\
= & \left(\begin{array}{c}
\hat{N} \\
k
\end{array}\right)\left(1-p_{b}^{j-1}\right)\left(P\left\{C^{j-1}\right\}\right)^{\hat{N}-k} \prod_{i=0}^{N B_{\max }}\left(1-P\left\{S_{i}^{j-1}\right\}\right)^{k},
\end{aligned}
$$

where $\left(1-p_{b}^{j-1}\right)\left(P\left\{C^{j-1}\right\}\right)^{\hat{N}-k}$ is the probability that $\hat{N}-k$ nodes transmit in $j$ and $\prod_{i=0}^{N B_{\max }}\left(1-P\left\{S_{i}^{j-1}\right\}\right)^{k}$ is the probability that the remaining $\mathrm{k}$ nodes do not transmit, since they do not sense the channel in slot $j-1 . N_{c}^{j-1}$ of eq. (9) is, on its turn, a random variable, binomially distributed, having a probability distribution which depends on the probabilities $P\left\{T^{l}\right\}$ with $l \in(1, . ., j-2)$. By increasing the initial number of nodes in the network, $N_{c}^{0}$, and the time slot considered (i.e., the value of $j$ ), the complexity of the evaluation of the statistics of $N_{c}^{j-1}$ increases exponentially, because of the need to follow all possible combinations of values of $N_{c}^{0}, N_{c}^{1}$, etc. To reduce this complexity we introduce an approximation: we do not model $N_{c}^{j-1}$ as a random variable, but we set its value at the initial value of $N_{c}$, that is $N_{c}^{0}=N$. In the following we will denote as $N_{c}$ (equal to $N$ ), the number of competitors whatever the slot $j$. In Section VI the mathematical model results are compared with simulation results: the Figures show that this approximation does not affect very much performance. Moreover, in [6] it is shown that results obtained by modelling $N_{c}^{j}$ according to its distribution, and the ones obtained with the above defined approximation are roughly the same for all the scenarios studied. 
Now, we can evaluate the probability, $p_{s}$, that a generic packet is transmitted with success on the channel, given by:

$$
p_{s}=\sum_{j=0}^{t_{\max }} P\left\{Z^{j}\right\}
$$

where $P\left\{Z^{j}\right\}$ is the probability that, in slot $j$, one and only one transmission occurs, which means that the packet is transmitted with success. This occurs when, being the channel free in slot $j-1$ (which occurs with probability $\left(1-p_{b}^{j-1}\right)$ ), one and only one node, over $N_{c}$, senses the channel in slot $j-1$. Therefore, it is given by:

$P\left\{Z^{j}\right\}=\left(1-p_{b}^{j-1}\right) P\left\{C^{j-1}\right\} \cdot \prod_{i=0}^{N B_{\max }}\left(1-P\left\{S_{i}^{j-1}\right\}\right)^{N_{c}-1}$

where $P\left\{C^{j-1}\right\}$ is the probability that one node senses the channel in $j-1$ and $\prod_{i=0}^{N B_{\max }}\left(1-P\left\{S_{i}^{j-1}\right\}\right)^{N_{c}-1}$ is the probability that the remaining $N_{c}-1$ nodes do not sense the channel in slot $j-1$.

\section{Energy consumption}

Now, let us derive the mean energy consumed by a node during a round. A node spends energy when it receives or transmits a packet and also when it is in backoff state. After the transmission of the packet, the node switches off and does not consume energy. The node will stay in the off state till the reception of the following query.

We set as $P_{s}=82.5 \mathrm{~mW}$ the power spent in receiving and sensing states; $P_{i}=50 \mathrm{~mW}$ the power spent in backoff state and $P_{t}=75.8 \mathrm{~mW}$ the power spent in transmission state. These values are taken from data sheets of Freescale devices [13]. The mean energy spent by a node in a round, is given by:

$$
E_{\text {mean }}=\sum_{j=0}^{t_{\max }} E_{t}^{j}+E_{s}^{j}+E_{i}^{j}
$$

where $E_{t}^{j}, E_{s}^{j}$, and $E_{i}^{j}$ are the energies spent in transmission, sensing and backoff, respectively, for a node which transmits its packet in slot $j$.

Since no retransmissions are performed, each node will transmit only one packet per round. Therefore,

$$
E_{t}^{j}=P_{t} \cdot \frac{N_{b i t}}{R_{b}} \cdot P\left\{T^{j}\right\}
$$

where $N_{\text {bit }}$ is the packet size, equal to 10 Bytes; $R_{b}$ is the bit rate, equal to $250 \mathrm{kbit} / \mathrm{sec}$, and $P\left\{T^{j}\right\}$ is the probability that the node transmits its packet in slot $j$.

The energy spent in sensing state depends on the number of slots in which the node senses the channel. A node that transmits in slot $j$ could have sensed the channel for one slot, in case it has found the channel free at the end of the first backoff stage, two slots in case it found the channel free at the end of the second backoff stage, and so on. This energy is given by:

$$
E_{s}^{j}=P_{s} \cdot \frac{N_{b i t}}{R_{b}} \cdot\left(1-p_{b}^{j-1}\right) \sum_{k=0}^{N B_{\max }}(k+1) \cdot P\left\{S_{k}^{j-1}\right\},
$$

where $\left(1-p_{b}^{j-1}\right) \cdot P\left\{S_{k}^{j-1}\right\}$ is the probability that a node that is at the end of the $k$-th backoff stage finds the channel free and transmits.

Finally, the energy spent in the backoff state depends on the number of slots in which the node does backoff. This number depends on the number backoff stages performed. Therefore,

$$
E_{i}^{j}=P_{i} \cdot \frac{N_{b i t}}{R_{b}} \cdot\left(1-p_{b}^{j-1}\right) \sum_{k=0}^{N B_{\max }}(j-k-1) \cdot P\left\{S_{k}^{j-1}\right\},
$$

where $(j-k-1)$ is the number of slots in which a node which transmits in slot $j$ and has performed the $k$-th backoff stage, does backoff. This value is the same whatever the values of backoff counter extracted in each backoff stage.

\section{Derivation of the probability that the channel is found busy}

The probability that a node, at the end of the sensing phase, finds the channel busy, in a given slot $j$, is the probability that in that slot one or more nodes transmit their packets. This probability is given by:

$$
p_{b}^{j}=1-\left[p_{b}^{j-1}+\left(1-p_{b}^{j-1}\right) \prod_{i=0}^{N B_{\max }}\left(1-P\left\{S_{i}^{j-1}\right\}\right)^{N_{c}-1}\right]
$$

where the term between square brackets is the probability that the channel in slot $j$ is free. If the channel in slot $j-1$ is busy, no transmissions may occur in slot $j$; therefore slot $j$ will be certainly free. If, instead, the channel in $j-1$ is free, the channel in $j$ will be free only if there are not nodes sensing the channel in $j-1$.

\section{NUMERICAL RESULTS}

For the purpose of numerical comparison, a dedicated simulation tool, implementing the 802.15.4 CSMA/CA protocol described in Section II, has been developed. We consider $N$ nodes and a sink, sending queries and waiting for the data from nodes. Ideal channel conditions are assumed, therefore all nodes can "hear" each other and can receive correctly the query at each round. No capture effect is considered: in case two or more packets collide, they are all lost.

In the Figures 2 and 3 the probabilities $P\left\{T^{j}\right\}$ and $P\left\{Z^{j}\right\}$ as a function of time $t=j$ (representing the time slot from 0 to $\left.t_{\max }\right)$, are shown, respectively, for different values of $N$. In both Figures the backoff parameters are set to the values used in the 802.15.4 standard: $B E_{\min }=3, B E_{\max }=5$ and $N B_{\max }=4$. Both mathematical analysis and simulation results are reported. As we can see, there are no relevant differences between results; therefore, the model is validated. The differences are due to the approximation made in modelling $N_{c}^{j}$. 
In Figure 2 the cases $N=5$ and 10 are considered. In both cases, no traffic is present in the first slot, because no transmissions may occur: a node that extracts the 0 value at the first backoff stage will sense the channel in slot 0 and will transmit in slot 1 . This happens with probability $1 / W_{0}=1 / 8$ whatever $N$. When a node tries to access the channel for the first time, it will delay the transmission for a random number of slots in the range $\left[0, W_{0}-1\right]$. Since there are few nodes in the network, the probability to find the channel busy is low and, thus, the probabilities to transmit a packet in the first contention window (from 1 to $W_{0}$ ) are larger than the probabilities associated to the slots from $W_{0}+1$ to $W_{0}+W_{1}-1$. In these slots, in fact, only the nodes which have found the channel busy and are performing the second backoff stage, transmit (plus some nodes already performing the third of fourth of fifth backoff stage, which are a minority). For $j \geq W_{0}+W_{1}$ the probabilities decrease further, because here transmit only nodes that have finished the second backoff and are running the third or fourth or fifth backoff stage. As we can see, by increasing the number of nodes in the network the probabilities to have transmissions in slots from 2 to $W_{0}$ decrease and consequently the probabilities to have transmissions in slots from $W_{0}+1$ to $W_{0}+W_{1}-1$ increase. The reason is that by increasing the number of nodes the probability to find the channel busy at the end of the first backoff increases.

In Figure 3 the cases $N=3,6$ and 9, are shown. The trend is very similar to the one obtained for $P\left\{T^{j}\right\}$.

In the Figures described above is shown the traffic up to $t=$ $j=24$, because for larger values of $j$ the traffic is negligible: $P\left\{T^{j}\right\}$ and $P\left\{Z^{j}\right\}$ tend to zero.

In Figures $4,5 p_{s}$ and $E_{\text {mean }}$ are reported, respectively, for $N=2, . ., 10$, by varying the values of $W_{i}$, having fixed $N B_{\max }=4$. In particular, we consider the cases: absence of exponential backoff, that is $W_{i}=4,8,16$ and 32 whatever $i$ (which correspond to $B E_{\min }=B E_{\max }=2,3,4$ and 5); and exponential backoff obtained by setting $B E_{\max }=5$ and $B E_{\min }=2,3$ (the standard case) and 4 (which correspond to $W_{0}=4,8$ and 16). The symbols in the Figures (e.g., circles, squares, stars, etc..) are obtained through simulation, whereas the curves are obtained through the mathematical model.

As we can see in Figure 4, by increasing $W_{0}, p_{s}$ increases, because the probability that two or more nodes extract the same backoff counter decreases. Fixed $W_{0}$, the use of the exponential backoff improves performance. This improvement is more significant the larger is $N$ and the smaller is $W_{0}$.

As we can see the backoff algorithms leading to better performance in terms of reliability are less energy efficient. For example, the case $W_{i}=4$ is the best from the energy consumption viewpoint and the worst from the reliability viewpoint.

For low values of $N$ (for example $N=2$ ), if we pass from the standard values of $W_{i}$ to the case $W_{i}=32$, we obtain an improvement of $10 \%$ of $p_{s}$ but also a relevant worsening in energy consumption (the energy consumed is tripled). Therefore, it is not convenient. For large values of $N$ (for example $N=10$ ), instead, if we pass from the standard case to $W_{i}=32$, we obtain an improvement of almost $30 \%$ of $p_{s}$ while the energy is less than doubled; therefore, in some cases it could be a convenient option.

However, the choice of the backoff algorithm to be implemented depends on the application: in case energy efficiency is a more stringent requirement with respect to reliability, low values of $W_{0}$ must be considered; on the opposite, larger values of $W_{0}$ should be used in case reliability is the main stringent requirement. As we can see in the Figures, the protocol defined in the standard realized a good trade-off between all the requirements.

\section{CONCLUSIONS}

A novel mathematical model for the IEEE 802.15.4 MAC protocol, considering a non-beacon enabled WSN, where a star topology is established, is provided. The mathematical model allows the evaluation of the statistical distribution of the traffic generated by nodes toward the sink. The probability that a node succeeds when accessing the channel, the success probability for the transmission of a packet, and the mean energy spent by a node per round, have been evaluated.

The model does not suffer from the limitations shown by related work in the literature, even if an approximation is needed when estimating the number of nodes competing at each instant for the channel. The comparison of results obtained through simulations and the mathematical model shows that the latter captures the behavior of the medium access control mechanism, and well characterises the traffic generated regardless of the approximation mentioned.

Finally, a comparison between performance obtained with the protocol defined by the standard and other protocols obtained by varying some parameters characterising the backoff algorithm, is also provided. Results show that a tradeoff between energy efficiency and reliability must be found. The choice of the backoff algorithm to be implemented depends on the application. However, the protocol defined in the standard realizes a good trade-off between all the requirements.

\section{ACKNOWLEDGMENT}

This work has been supported by the EC-funded Networks of Excellence CRUISE and NEWCOM++.

\section{REFERENCES}

[1] IEEE 802.15.4: Wireless Medium Access Control (MAC) and Physical Layer (PHY) Specifications for Low-Rate Wireless Personal Area Networks (LR-WPANs), IEEE, 2003.

[2] I. F. Akyildiz, W. Su, Y. Sankarasubramaniam, E. Cayirci, "A Survey on Sensor Networks", IEEE Communications Magazine, Aug. 2002, 102114.

[3] M. Tubaishat, S. Madria, "Sensor Networks: an Overview", IEEE Potentials, Vol. 22 Issue 2, April/May 2003.

[4] The ZigBee Alliance web site: http://www.zigbee.org/en/index.asp

[5] A. Koubaa, M. Alves, E. Tovar, "Modeling and Worst-Case Dimensioning of Cluster-Tree Wireless Sensor Networks", 27th IEEE International RealTime Systems Symposium (RTSS'06), December 5-8, 2006, Rio de Janeiro, pp. 412-421.

[6] C. Buratti, R. Verdone, "Performance Analysis of IEEE 802.15.4 NonBeacon Enabled Mode", submitted to IEEE Transaction on Vehicular Technologies. 


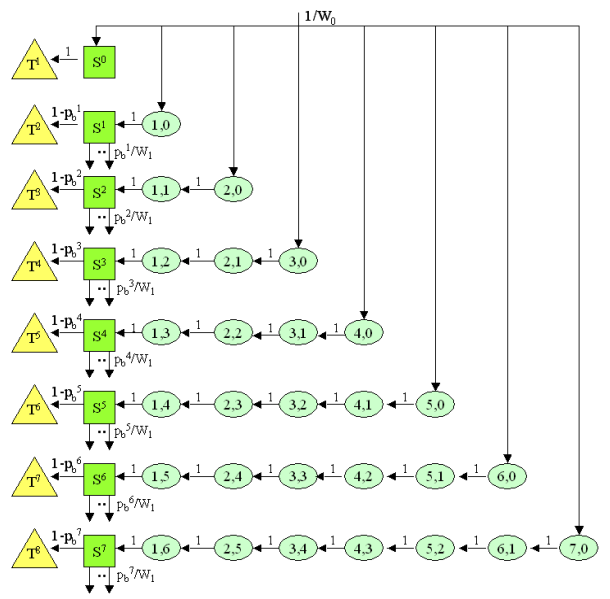

Fig. 1. The state transition diagram related to the first backoff stage $\left(B O_{s}=0\right)$.

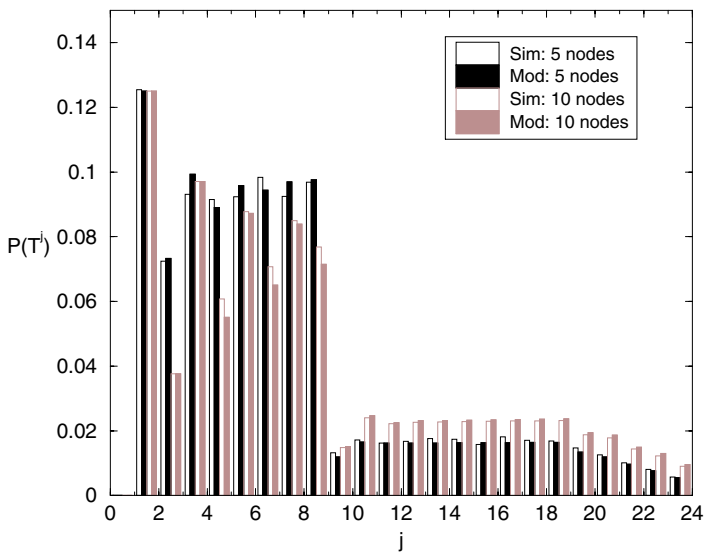

Fig. 2. The probabilities $P\left\{T^{j}\right\}$ obtained through simulation and through the mathematical model in the cases $N=5$, and 10 .

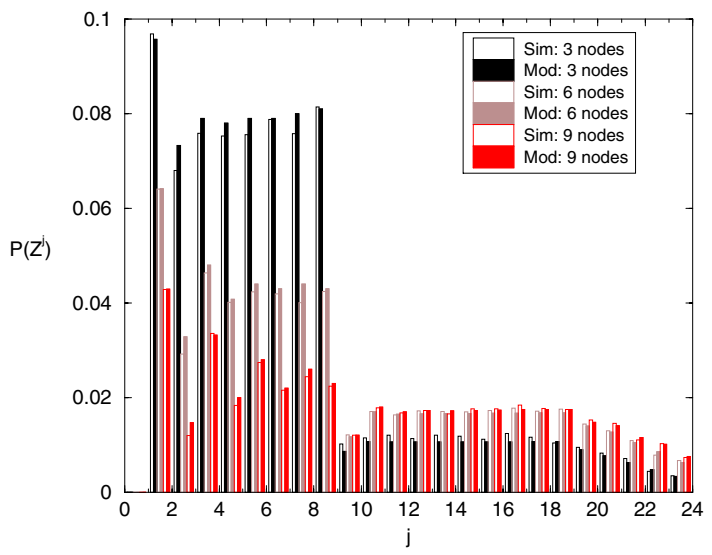

Fig. 3. The probabilities $P\left\{Z^{j}\right\}$ obtained through simulation and through the mathematical model in the cases $N=3,6$ and 9 .

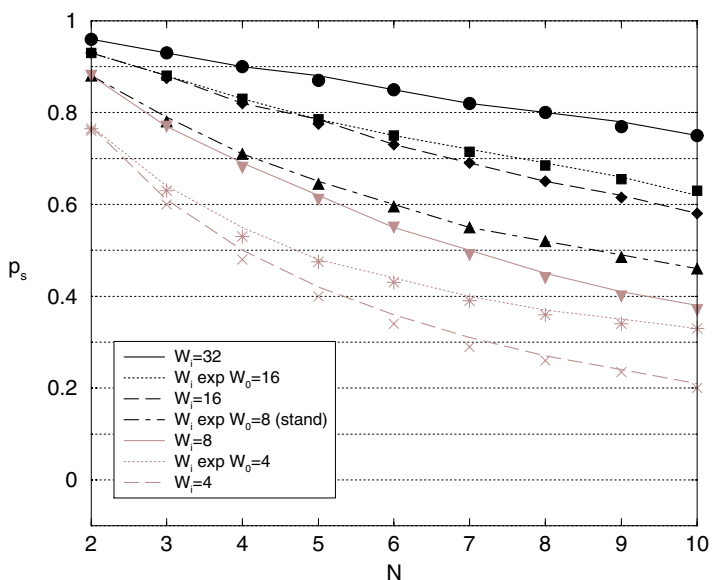

Fig. 4. The probability $p_{s}$ obtained through simulation (symbols) and through the mathematical model (lines) for different values of $N$, by varying the values of $W_{i}$, having fixed $N B_{\max }=4$.

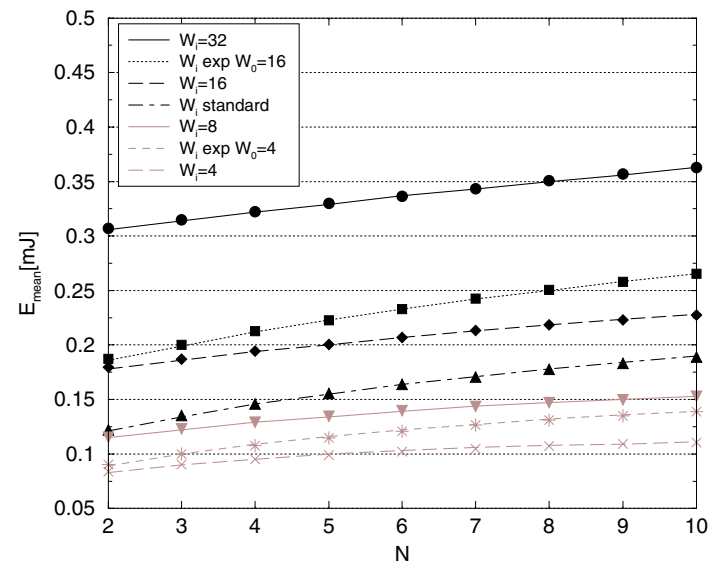

Fig. 5. The mean energy spent, $E_{\text {mean }}$ in $m J$ obtained through simulation (symbols) and through the mathematical model (lines) for different values of $N$, by varying the values of $W_{i}$, having fixed $N B_{\max }=4$.

[7] J. Misic, S. Shafi, V. B. Misic, "Maintaining Reliability Through Activity Management in an 802.15.4 Sensor Cluster," IEEE Transactions on Vehicular Technology, Vol. 55, No. 3, May 2006, pages: 779-788.

[8] S. Pollin, M. Ergen, S.C. Ergen, B. Bougard, L. Van der Pierre, F. Catthoor, I. Moerman, A. Bahai, P. Varaiya, "Performance Analysis of Slotted Carrier Sense IEEE 802.15.4 Medium Access Layer", Proceeding of GLOBECOM, 2006, San Francisco, California, November 27 December 1 .

[9] T.R. Park, T.H. Kim, J.Y. Choi, S. Choi and W.H. Kwon, "Throughput and energy consumption analysis of IEEE 802.15.4 slotted CSMA/CA", IEEE Electronics Letters, vol. 41, issue 18, pp. 1017-1019, Sept. 2005.

[10] T. O. Kim, H. Kim, J. Lee, J. S. Park, and B. D. Choi, "Performance Analysis of the IEEE 802.15.4 with Non-beacon Enabled CSMA/CA in Non-saturated Contition", EUC 2006, pp. 884-893, 2006.

[11] G. Bianchi, "Performance Analysis of the IEEE 802.11 Distributed Coordination Function", IEEE Journal on Selected Areas in Communications, Vol. 18, n. 3, March 2000.

[12] Leonard Kleinrock, "Queueing Systems", John Wiley and Sons, Inc. 1975.

[13] Moteiv Corporation Tmote Sky: Datasheet, June 2006, see the website: http://www.moteiv.com 